\title{
Online E-commerce Venture - Choose The Best
}

\author{
Prof. Vidya Zope \\ Professor, \\ Department of Computer Engineering, \\ Vivekanand Education Society's \\ Institute of Technology, \\ Maharashtra, India \\ vidya.zope@ves.ac.in \\ Drishti Parchani \\ Department of Computer Engineering, \\ Vivekanand Education Society's \\ Institute of Technology, \\ Maharashtra, India \\ 2017.drishti.parchani@ves.ac.in
}

\author{
Divya Borse \\ Student, \\ Department of Computer Engineering, \\ Vivekanand Education Society's \\ Institute of Technology, \\ Maharashtra, India \\ 2017.divya.borse@ves.ac.in \\ Suraj Gangwani \\ Department of Computer Engineering, \\ Vivekanand Education Society's \\ Institute of Technology, \\ Maharashtra, India \\ 2017.suraj.gangwani@ves.ac.in
}

\author{
Divya Kalwani \\ Department of Computer Engineering, \\ Vivekanand Education Society's \\ Institute of Technology, \\ Maharashtra, India \\ 2017.divya.kalwani@ves.ac.in
}

\begin{abstract}
This paper entitled 'E-commerce venture -choose the best' plays a key role in the upliftment of digitization and automation of small-scale businesses. The majority of the small businesses in India don't have a website according to the survey conducted by GoDaddy-world's largest technology services provider. In short, the proposed system aims at building online ecommerce ventures to satisfy the needs of small businesses and enterprises by providing extensive machine learning features.
\end{abstract}

Keywords:- Recommender system, Online business, Online system, Users, Data processing, Security, E-commerce.

\section{INTRODUCTION}

Technology is advancing at breakneck speed. By using Machine Learning we can take customer experience to a whole new level .

In today's competitive and convenience focused society, customers want to shop from their own homes,making ecommerce a flexible solution for both businesses and buyers.Small businesses as well as enterprises can be benefited hugely from their own ecommerce website.Most of India's small businesses are largely 'invisible' online or have a marginal digital footprint, according to a survey released by RedShift Research and GoDaddy, the world's largest technology provider dedicated to small businesses[5].

Average shopping cart abandonment rate is $68.81 \%$ and issues faced by customers are checkout flow and design.

Our system aims at building an easy to use and simple interface with secure payment options. Small businesses are lacking to provide the right message at the right time which can be overcome by personalization and product recommendation.

We have chosen this problem statement in order to make some contribution to satisfy the needs of small businesses and enterprises by providing online ecommerce venture with extensive machine learning features to help to increase online presence and sell products online to a large customer base.

Our project aims at developing an e-commerce website which will include various functionalities such as recommending the products that are highly in demand apart from this our system will provide an automation process which will take an existing e-commerce process to the whole new level from not only a customers aspect but from seller's aspect as well .Starting from the customer placing an order and then seller being notified about the details till finally the customer placing a feedback about the system everything will be automated.To enhance the user experience we are also planning to add few machine learning algorithms for inducing the functionalities such as a best selling product based on the past experience and inventory prediction functionality that would help the seller for updating his inventory.

\section{RELATED WORKS}

\section{A. Product Recommendation Techniques for Ecommerce - past, present and future [2]:}

It Outlines Product recommendation techniques which are being used widely to recommend the scrutinized product to the customers which consists of overview of recommendation techniques with suitable examples and illustrative diagrams.Paper consists of detailing of algorithms used to build recommendation systems but it lacks actual implementation details.

\section{B. Developing an E-commerce website [1]:}

This paper outlines different aspects of developing an ecommerce website which involves a detailed planning process with use case, architectural pattern of the web application and the optimum solution to the challenges involved in developing one.But it lacks automation process as paper consists of detailing of building traditional e-commerce systems without machine learning features.

\section{A Review Paper on E-Commerce [3]:}

The objectives of study are: 1 . To understand the present status and trends of E-Commerce; and 2. To reveal the key variables influencing the increased usage of E-Commerce.The paper also focused on the change in the E-commerce business from last one decade, which has impacted the business of the retail stores and listed some the implications on the businesses with the solutions to various issues which will benefit the 
growth in E-commerce by 2020.Paper focuses on current trends of e-commerce but lacks detailing of each trend.

\section{Product Recommendation Systems a Comprehensive Review [4]:}

Paper focused on formulation of decision making either by the use of collaborative filtering or by the use of content filtering in order to build a personalized recommendation system.

Paper has proper elaboration about content based filtering and collaborative filtering but it doesn't highlight implementation challenges faced while building the recommendation system.

\section{IMPLEMENTATION DETAILS}

In this research, an online e-commerce venture for beauty products is proposed which allows users to purchase beauty products online. This paper is not only focusing on how to place an order to buy beauty products but also aiming at transitioning traditional systems into automated systems.our system will provide an automation process which will take an existing e-commerce process to the whole new level from not only a customers aspect but from seller's aspect as well.Starting from the customer placing an order and then seller being notified about the details till finally the customer placing a feedback about the system everything will be automated.To enhance the customer's experience,we are also providing extensive machine learning features such as product recommendation based on purchase history of the customer,product recommendation based on user features, product recommendation based on user's favorites, recommending top-rated products,data visualization etc.

\section{So,the basic features of the proposed product are:}

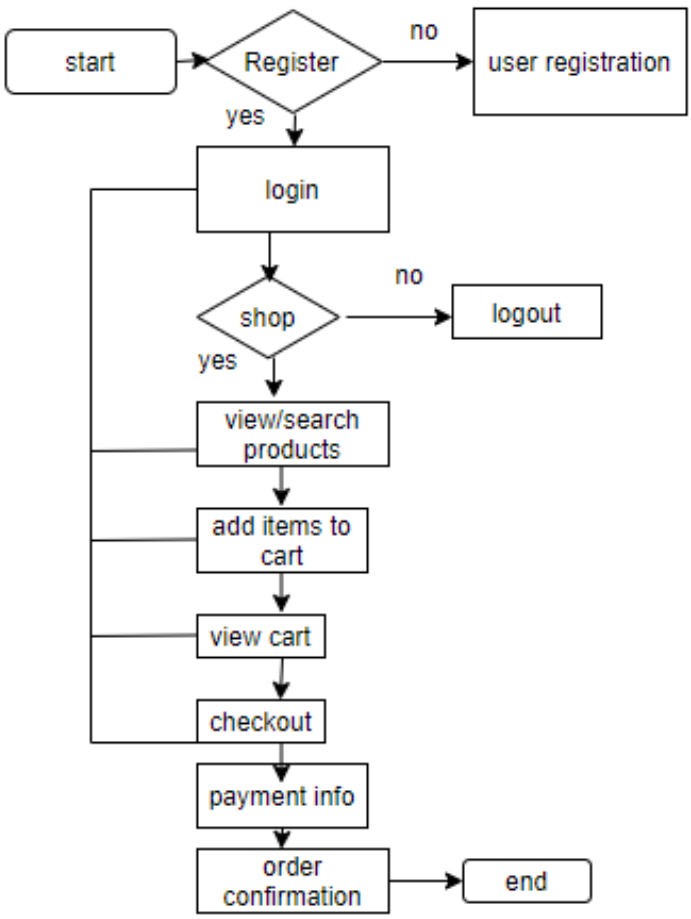

Fig. 1. Block Diagram for user side functionality of e-commerce website

\section{A. User side functionality:}

1) User login/Sign up functionality:

In order to purchase the product the user has to sign in if he/she is not already a registered user otherwise the user can proceed with sign up functionality.

\section{2) Product navigation:}

After logging in, users can view various beauty products along with their description, images, price, ingredients information in order to decide which product to purchase.

3) Add to cart:

We can add favorite products in the cart so that in future if we want to buy them,we can use the view cart option.

4) Search:

To search for specific beauty products.

5) Product view history:

Displays all the products viewed by the user since his/her logging.

6) Contact us:

To contact admin in case of some query

7) Payment option:

Secure payment mechanism

8) About us:

To show information about online e-commerce beauty product ventures.

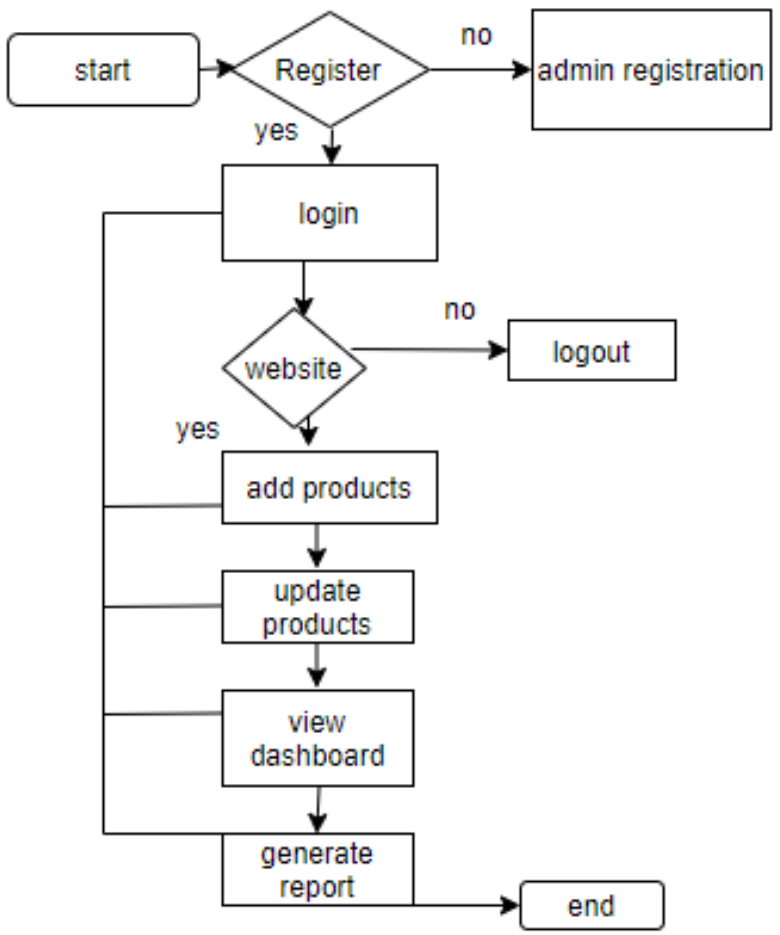

Fig. 2. Block Diagram for admin side functionality of e-commerce website 


\section{B. Admin side functionality:}

1) Admin login/Sign up functionality:

In order to access information such as products bought by users,total sales, add new products,remove products,admin has to logged in otherwise he/she has to sign in to register himself/herself as an Admin.

\section{2) Dashboard:}

Admin can view the purchase rate,products like by user's,monthly sale,yearly sale in the form of graphs,pie chart.In shot admin will have a dashboard to get information about above topics.

3) Add beauty product:

Admin can add products into the website along with price, description, images and other useful information.

\section{Integrations with Django:}

\section{1) MailChimp integration:}

This MailChimp Integration connects with our customer through email for that customer needs to subscribe. As he subscribes He would receive future offers through the mail.

\section{2) Stripe Integration:}

The Stripe Integration is used to make the payments safer.It is used to store the payment details while storing it in the card would be risky and can result in data leak and various other problems. But Stripe integration makes it safer by storing it onto the stripe cloud.

\section{Advanced extensive machine learning features:}

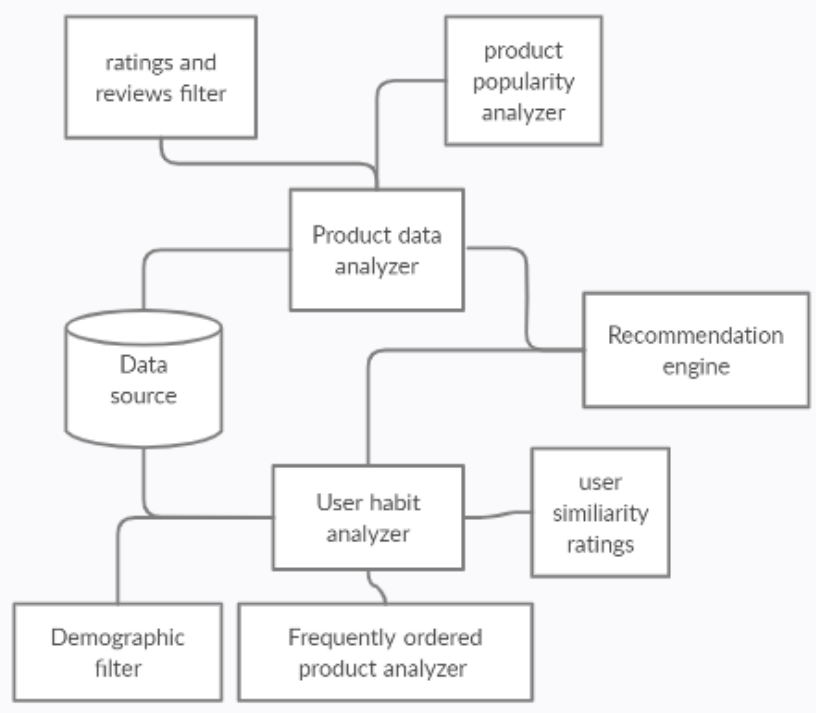

Fig. 3. Block Diagram for product recommendation system

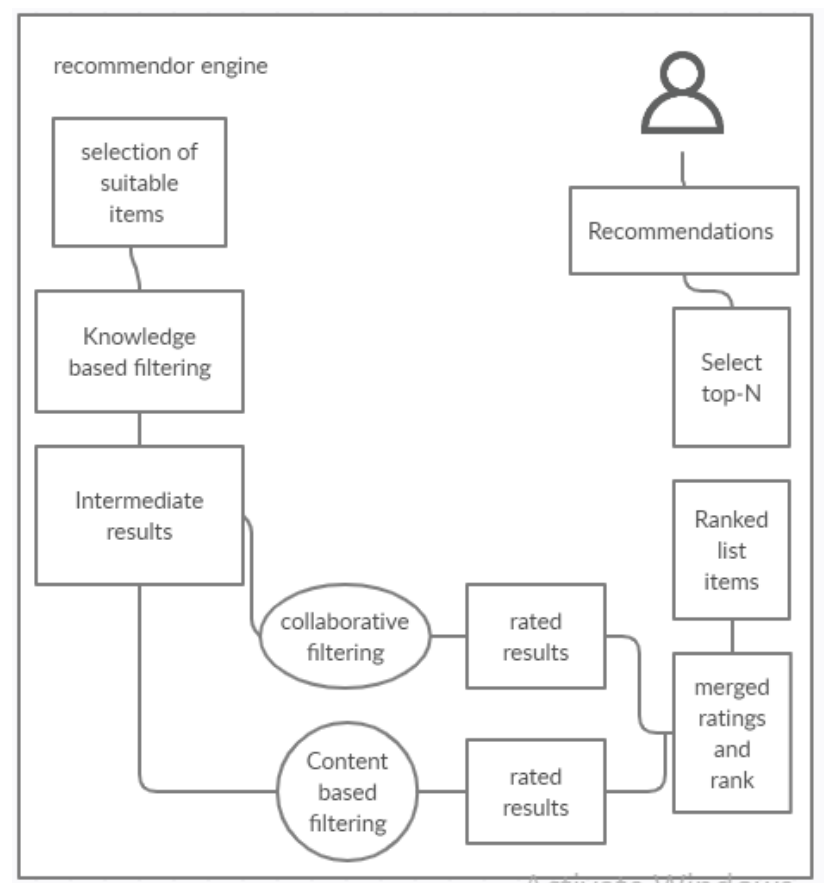

Fig. 4. Block Diagram for recommendation engine

\section{Product recommendation based on user features:}

Whenever we want to try new beauty products,it's difficult to choose.It's sometimes scary because new products you have chosen might not be suitable for your skin.So proposed system is aimed at building skincare product recommendation system.We have taken dataset from kaggle which has information about the brand, the price, the rank, skin types and the chemical components of each item.It takes user features such as skin tone,skin type,eye color,hair color etc and based on that recommends the product which are made for specific skin type.

For example,a person having tan skin type will get recommendations about the products which are suitable for that specific skin.

In short,the product recommendations can be based on textual clustering analysis given in the product description.

\section{Steps to build recommendation system:}

1) Dataset:

For this project,we have used a Skin care dataset available on Kaggle.It has a total 8650 entries with 25 columns.Column names as follows:

\begin{tabular}{|l|l|l|l|l|}
\hline Username & Skin type & Eye color & Hair color & Rating star \\
\hline Review & Product & Brand & Price & Rating \\
\hline Ingredients & combination & Dry & normal & oily \\
\hline Sensitive & category & product_url & user_id & prodcut_id \\
\hline $\begin{array}{l}\text { Ingredients } \\
\text { _Celaned }\end{array}$ & $\begin{array}{l}\text { review_clean } \\
\text { ed }\end{array}$ & good_stuff & ing_tfidf & Skin tone \\
\hline
\end{tabular}


2) Data preprocessing steps:

To propose a system like this, we have used a dataset which is available in kaggle. We have removed duplicate entries in the dataset.

3) After performing preprocessing operations on datasets, we have done the data visualization part in order to understand the nature of available data.

In the first recommendation part,we are suggesting user products based on their features.

It means if one user shares skin type, tone, eye color, hair color, with another user, there is a chance they will enjoy the same products.For that we are analyzing in terms of skin type,skin tone,eye color,hair color,rating stars.
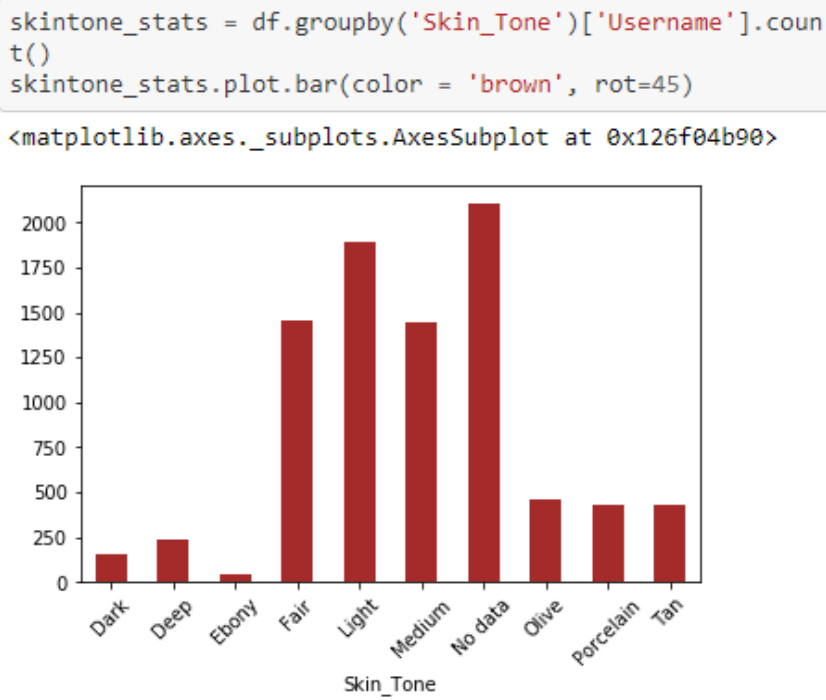

Fig. 5. Graph to show relationship between user and skin tone

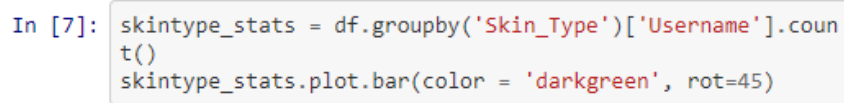

Out [7]: 〈matplotlib.axes._subplots.AxesSubplot at 0x124dad490〉

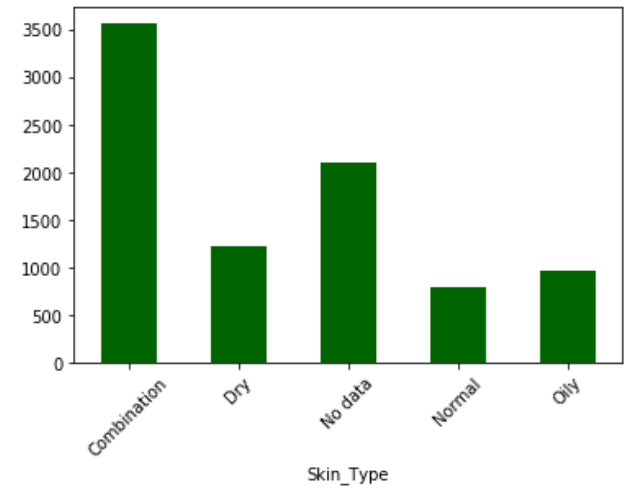

Fig. 6. Graph to show relationship between skin type and user
In [8]: eyecolor_stats $=d f$.groupby ('Eye_Color') ['Username'].coun $t()$

eyecolor_stats.plot.bar(color $=$ 'darkblue', rot $=45)$

Out [8]: 〈matplotlib.axes._subplots.AxesSubplot at 0x124d17610〉

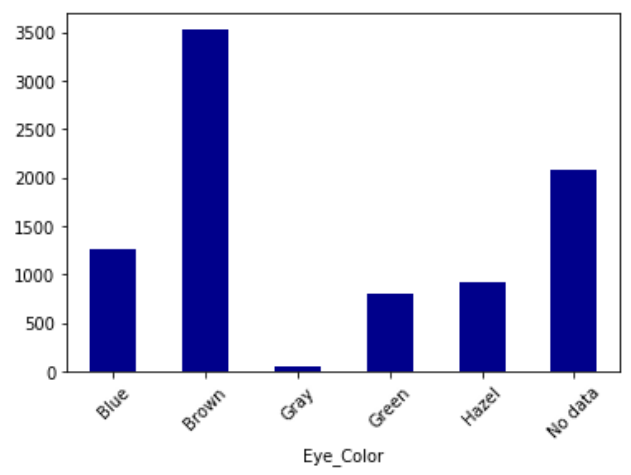

Fig. 7. Graph to show relationship between eye color and user

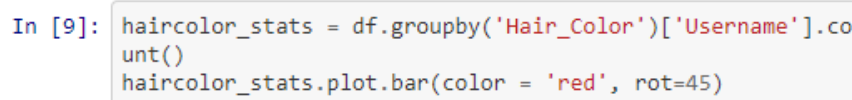

In [9]: haircolor_stats $=\mathrm{df}$.groupby ('Hair_Color') ['Username'].co unt()

haircolor_stats.plot.bar $($ color $=$ 'red', rot $=45)$

Out[9]: 〈matplotlib.axes._subplots.AxesSubplot at $0 \times 124$ e9bd10〉

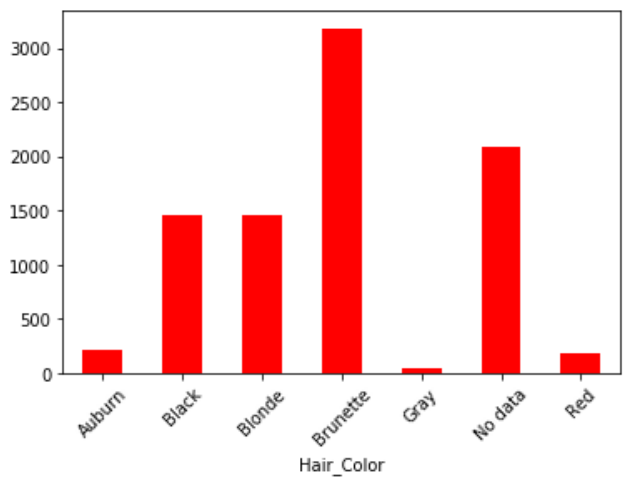

Fig. 8. Graph to show relationship between hair color and user

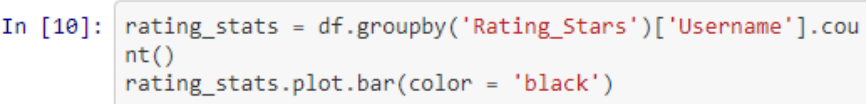

In [10]: rating_stats $=$ df.groupby ('Rating_Stars') ['Username'].cou nt () rating_stats.plot.bar (color = 'black')

Out[10]: 〈matplotlib.axes._subplots.AxesSubplot at $0 \times 124 \mathrm{fbd} 410$ >

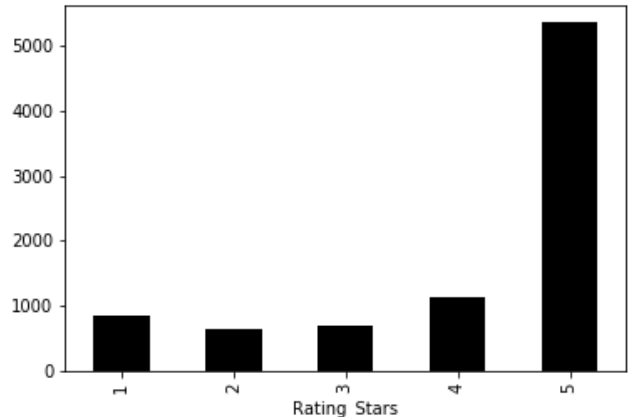

Fig. 9. Graph to show relationship between ratings and user 
This gives a glimpse of different skin features of different user. It makes it easy to recommend products to the user who share similar features.

1. We have used collaborative filtering algorithms. Two types of collaborative filtering:

1. User-based collaborative filtering: predict the items that user might like based on the rating given by other users who share similar taste with the target user.

\section{Item-based collaborative filtering:}

It explores the relationship between the pair of items.

2. We have used user-based collaborative filtering because if one user shares skin type, tone, eye color, hair color, with another user, there is a chance they will enjoy the same products.

3. Finding the similarity of users to the target user U.Similarity for any two users ' $x$ ' and ' $y$ ' can be calculated from the given formula:

$\operatorname{Cos}(\mathbf{x}, \mathbf{y})=\mathbf{x} \cdot \mathbf{y} /\|\mathbf{x}\| *\|\mathbf{y}\|$.

$\mathrm{x} \cdot \mathrm{y}=$ product (dot) of the vectors ' $\mathrm{x}$ ' and ' $\mathrm{y}$ '. $\|\mathrm{x}\|$ and

$\|y\|=$ length of the two vectors ' $x$ ' and ' $y$ '.

$\|\mathrm{x}\| *\|\mathrm{y}\|=$ cross product of the two vectors.

We have formed interaction matrix which consists of user_id and product_id.To create this matrix,we have filled entire matrix with zeros initially. The length of the matrix is the total number of products. The width of the matrix is the total number of users.We have filled the matrix either with 1 or 0. If user is associated with the product then fill that corresponding column with 1 otherwise fill it with 0 .

4. To achieve our target,we have compared skin features which we have taken as an input to the skin features of all the existing users features present in the dataset.After comparing,we will get new set of user's who matches with user's features. Then we can get products purchased by those users in order to recommend to user who has inputted his/her skin features. We will filter the products on the basis of ratings and only top rated products will be recommended to the user.

\begin{tabular}{l} 
Recommendation system \\
Skin Tone \\
Dark \\
Skin Type \\
Normal \\
Eye color \\
Brown \\
Hair color \\
\hline Black $\quad$ Product \\
Based on your features, these are the top products for you: \\
Ratings $\quad$ Mattifying Blotting Films \\
\hline 5 \\
\hline 5
\end{tabular}

\section{Product recommendation based on user's favorites:}

This feature is good for opinionated and experienced users.It gives meaningful recommendations to the user's favorite product based on similarity of the ingredients.

There are many algorithms used for building product recommendation system.Mostly used methods are collaborative filtering and content-based filtering.

In this project, we have applied content based recommendation for two reason:

1. Actually it is inappropriate to predict the beauty products based on user's past purchase history.That's because past products are much smaller than the test data.Predicting products based on past experience becomes difficult and does not give guarantee result because user must have tried some cosmetics but number of cosmetic products in the world are much larger than that.

2. There could be some people who have very similar taste.So here we can use user-user collaborative filtering to recommend new cosmetic products based on ranking values on its neighbouring groups.But every person has different skin type and feature so it's become a very ticky problem to recommend the right product to the user. So to get reliability and stability in the recommendation we need to focus on ingredients of each product and get similarities based on them.

Result: 
Steps:

1. Data preprocessing steps are similar to the first method.

2. We have applied natural language processing concepts to ingredients in order to find similarity between inputted product and products in the dataset.

To get to our end goal of comparing ingredients in each product, we first need to do some preprocessing tasks and bookkeeping of the actual words in each product's ingredients list.The first step will be tokenizing the list of ingredients in the Ingredients column.

3. In this, we are using cosine similarity as a similarity measure.Basically similarity measure refers to distance with dimensions representing features of the data object, in a dataset.If distance is less, the degree of similarity is high and if distance is more, the degree of similarity is low.

4. Cosine similarity is a metric, helpful in determining how similar the data objects are irrespective of their size. We can measure the similarity between two sentences in Python using Cosine Similarity. In cosine similarity, data objects in a dataset are treated as a vector. The formula to find the cosine similarity between two vectors is -

$\operatorname{Cos}(\mathrm{x}, \mathrm{y})=\mathrm{x} \cdot \mathrm{y} /\|\mathrm{x}\| *\|\mathrm{y}\|$

$x \cdot y=$ product (dot) of the vectors ' $x$ ' and ' $y$ '.

$\|x\|$ and $\|y\|=$ length of the two vectors ' $x$ ' and ' $y$ '.

$\|\mathrm{x}\| *\|\mathrm{y}\|=$ cross product of the two vectors ' $\mathrm{x}$ ' and 'y'.

Here, we take product name as an user input and compare it's property with the remaining product. We sort the result set in ascending order to get top closest products.

5. Here, we take product name as an user input and compare it's property with the remaining product. We sort the result set in ascending order to get top closest products.

\section{Product recommendation based on brand's:}

Different users have liking for different brands. They prefer products of certain brands. That's why brandwise recommendation is added in the system.User has to select particular brand name and recommendation engine will suggest different products belong to that brand.

\section{Data visualization:}

With the help of pandas, matplotlib libraries we can make the admin's dashboard more interactive and easily interpretable. Proposed system is aimed at upliftment of online e-commerce system and to achieve same analysis of products purchased by users,monthly sales done, yearly sales done etc features will be used in order to take proper business decisions.

\section{FUTURE SCOPE}

- Intelligent chatbots

chatbots use natural language to communicate with customers, identify an issue and resolve the issue thereby we can enhance the quality of e-commerce service[6].

\section{- Fraud detection feature can be added}

Machine learning has the capability to process repetitive data at rapid speed and prevent fraud transactions before they happen[6].

- Digital Marketing can be used for promoting services offered by the system and reaching a maximum number of users..

- We can integrate with small companies and enterprises to provide them quality services as proposed by the system and make them adaptable at any environment .

\section{CONCLUSION}

Our system is committed to help small businesses and enterprises to grow in terms of sales and reach new customers.

\section{System is guided by $\mathbf{3}$ principles:}

customer obsession rather than competitor focus, commitment to operational excellence and long term thinking. It is a user-friendly, cost efficient system, implemented by keeping in mind the ease of the user. It is an attempt to bridge the gap between customers and product sellers and also trying to automate the process of selling and buying.

\section{ACKNOWLEDGMENT}

We wish to express a true sense of gratitude towards our Mentor Mrs Vidya Zope for giving us this opportunity. We would like to extend our gratitude to all team members for their time-to-time support and hard work because of which we are able to build well-written paper. We would also love to express our gratitude towards our family and friends.And also, With all respect and gratitude, we appreciate our success to the writers of reference papers that are referred by us in completion of this paper work activity which will be useful in presenting our survey paper.

\section{REFERENCES}

[1] Syed Emdad Ullah,Tania Alauddin,Developing an E-commerce website - IEEE Conference Publication, 2016

[2] Dr. Shahid Amin Bhat,Keshav Kansana,A Review Paper on E-Commerce, 2016

[3] Jatinder Kaur,S.K. Gupta,Rajeev Kumar Bedi,Product Recommendation Systems a Comprehensive Review , 2018

[4] Shahab Sohail,Rashid Ali,Product Recommendation Techniques for Ecommerce - past, present and future, 2012

[5] Majority of Small Businesses in India Don't Have a Website: GoDaddy Survey Reports

[6] Machine Learning For Ecommerce: How Does it Work?

[7] Towards Data Science Machine learning basics with-the k-nearest neighbors-algorithm.

[8] The best Machine Learning Use Cases in Ecommerce 